\title{
Influence of temperature and voltage on electrochemical reduction of graphene oxide
}

\author{
XIUQIANG LI, DONG ZHANG*, PEIYING ZHU and CHAO YANG \\ Key Laboratory of Advanced Civil Engineering Materials, Ministry of Education, College of Materials Science \\ and Engineering, Tongji University, 4800 CaoAn Road, Shanghai 200092, China
}

MS received 24 March 2013; revised 7 May 2013

\begin{abstract}
In this paper, the influence of temperature and voltage on direct electrochemical reduction were discussed in detail. Reduced graphene oxide is characterized with X-ray diffraction (XRD), fourier transform infrared spectroscopy (FT-IR) and field emission scanning electron microscopy (FE-SEM). It is found that the reduction degree of graphene oxide (GO) decreases gradually with the increase of applied temperature. The optimal applied temperature found in our experiment is $20^{\circ} \mathrm{C}$; Meanwhile, as the applied voltage increases from 0.1 to $12.5 \mathrm{~V}$, the reduction degree of graphene oxide increases gradually. However, above $2 \cdot 5 \mathrm{~V}$, increasing voltage has little effect on the reduction degree of graphene oxide.
\end{abstract}

Keywords. Graphene oxide; electrochemical reduction; voltage; temperature.

\section{Introduction}

Graphene, a single atomic layer of $s p^{2}$-bonded carbon atoms arranged in a honeycomb lattice, exhibits remarkable electronic, thermal and mechanical properties (Chae et al 2004; Balandin et al 2008; Lee et al 2008). A number of approaches have been employed to synthesize graphene, such as the micro-mechanical exfoliation of graphite, epitaxial growth, chemical vapour deposition and the reduction of graphene oxide. Among these methods, reduction of graphene oxide method stands out, since it is promising for the mass production of graphene-based materials. However, during the oxidation process, graphite powders are exfoliated and broken into layers with increased interlayer distance; the landscape of $s p^{2}$ carbon network is modified with oxygen-containing functional groups and defects, which decrease conductivity and thermal conductivity of GO (Cai 2008). Reduction can be tuned and partially recovered to produce electrically conducting material. Recently, methods of reduction are mainly listed as follows: thermal treatment (Yang and Zhou 2012), chemical reduction (Muszynski et al 2008; Liu and Chen 2012), ultraviolet excitation (Williams and Seger 2008; Akhavan and Ghaderi 2009) and electrochemical reduction (Wang et al 2009).

As well-known, direct electrochemical reduction for reducing graphene oxide without harsh and toxic chemicals, has the potential for rapid, large-area and environmental friendly production of films composed of paperlike screens. Although, Sung and Yan (2009) used the

*Author for correspondence (zhangdng@tongji.edu.cn) electrophoretic deposition (EPD) to reduce GO and obtained EPD-GO deposited films, in the condition of its thickness was $4 \mu \mathrm{m}$, the conductivity of GO obtained was $1.43 \times 10^{4} \mathrm{~s} \mathrm{~m}^{-1}$. However, the study lacks research on experimental conditions, especially the influence of temperature and voltage. Unsuitable temperature and voltage not only waste energy sources, but also influence reduction effect. Thus, the influences of temperature and voltage on direct electrochemical reduction will be discussed.

\section{Experimental}

\subsection{Materials}

Natural graphite $(30 \mu \mathrm{m})$, sulfuric acid (98\%), potassium permanganate (GR), hydrogen peroxide (30\%), hydrochloric acid (AR), copper electrode, FTO electrode, were purchased from Sinopharm Chemical Reagent Co., Ltd, China. They were of analytical grade and used as received.

\subsection{Procedure}

GO used in this study was synthesized from purified natural graphite by the modified Hummers method (Jeong et al 2008; Guo 2009). GO was dispersed in water and sonicated for $1 \mathrm{~h}$ at room temperature. After the preparation of colloidal suspensions of individual GO platelets in purified water, FTO and $\mathrm{Cu}$ were selected for anode and cathode, respectively. The distance between the electrode was $1.2 \mathrm{~cm}$, typical concentrations and reduction time of GO were $1 \mathrm{mg} / \mathrm{mL}$ and $30 \mathrm{~min}$. The temperature and voltage were controlled through a water bath and d.c. 
power. The GO platelets are negatively charged, due to the abundant deprotonated carboxylate groups and are thus electrophoretically drawn to the anode electrode. No electrochemical reactions occur, until electronic contact is made to the anode. Once electronic contact is made to the anode, electrons will move away from the platelets, causing oxidation of many of the carboxylate groups on the periphery of the platelets. All of the unpaired electrons formed by the Kolbe-like loss of $\mathrm{CO}_{2}$ are then free to migrate through GO framework, to find other unpaired electrons and form covalent bonds. The reaction of radicals to form two-electron bonds occurs mostly within the graphene platelets (Sung and Yan 2009). After deposition, samples were air-dried at room temperature for $24 \mathrm{~h}$.

\subsection{Characterization}

XRD was performed by using a D 8 advance X-ray diffractometer with $\mathrm{CuK} \alpha$ radiation $(\lambda=1 \times 5418 \AA$, Bruker, Germany). XRD test was operated in a $2 \theta$ range of $3-70^{\circ}$ as a continuous scan rate of $5 \% \mathrm{~min}$. Graphite's interlayer distance is about $0.4 \mathrm{~nm}\left(2 \theta=26^{\circ}\right)$. After oxidation, the interlayer distance increases to $0.9 \mathrm{~nm}\left(2 \theta=10^{\circ}\right)$, due to the formation of $-\mathrm{OH},-\mathrm{COOH}, \mathrm{C}=\mathrm{O}$ and $\mathrm{C}-\mathrm{O}-\mathrm{C}$. FT-IR spectrum was recorded at room temperature with attenuated total reflection (ATR) mode. The spectrum of graphite oxide illustrates the presence of carboxyl $\left(v_{\mathrm{C}=\mathrm{O}}\right.$ at $\left.1720 \mathrm{~cm}^{-1}\right)$, hydroxyl $\left(v_{-\mathrm{OH}}\right.$ at 3440 and $\left.1395 \mathrm{~cm}^{-1}\right)$ and epoxy $\left(v_{\mathrm{C}-\mathrm{O}-\mathrm{C}}\right.$ at $\left.1054 \mathrm{~cm}^{-1}\right)$. Most of the chemical groups were introduced during the oxidation process. Field emission scanning electron microscope (FE-SEM) images of reduction graphene oxide were obtained on a Gemini LEO1525 at $5 \mathrm{kV}$ in inlens detection mode. The sheet resistances of the films were measured by a Resistance Meter (SZT-2A, China) using a four-point probe head with a pin-distance of about $1 \mathrm{~mm}$. To reduce the contact resistance between the probes and film surface, the four contact points were coated with silver paste.

\section{Results and discussion}

\subsection{Influence of reduction voltage}

Compared with the pristine graphite, the characteristic peak of GO appeared at $10^{\circ}(002)$. AB stacking order in GO could still be observed and the layer-to-layer distance (d-spacing) was $0.849 \mathrm{~nm}$ (Jeong et al 2008). This value was larger than the $d$-spacing $(0.335 \mathrm{~nm})$ of pristine graphite $\left(2 \theta=26^{\circ}\right)$ due to the intercalated water molecules between layers. When GO was electrochemically reduced, the characteristic peak of graphite oxide (002) nearby $2 \theta=10^{\circ}$ absolutely disappeared and the characteristic peak of electrochemical reduction graphene oxide (ERGO) appeared at $26^{\circ}$ (Buchsteiner et al 2006). XRD spectra of ERGO obtained from electrochemical reduction at different voltages are shown in figure 1(a). The sharp diffraction peaks at $2 \theta=\sim 34^{\circ}(101), \sim 38^{\circ}$ (200) come from FTO. When applied voltages are $0 \cdot 1$ and
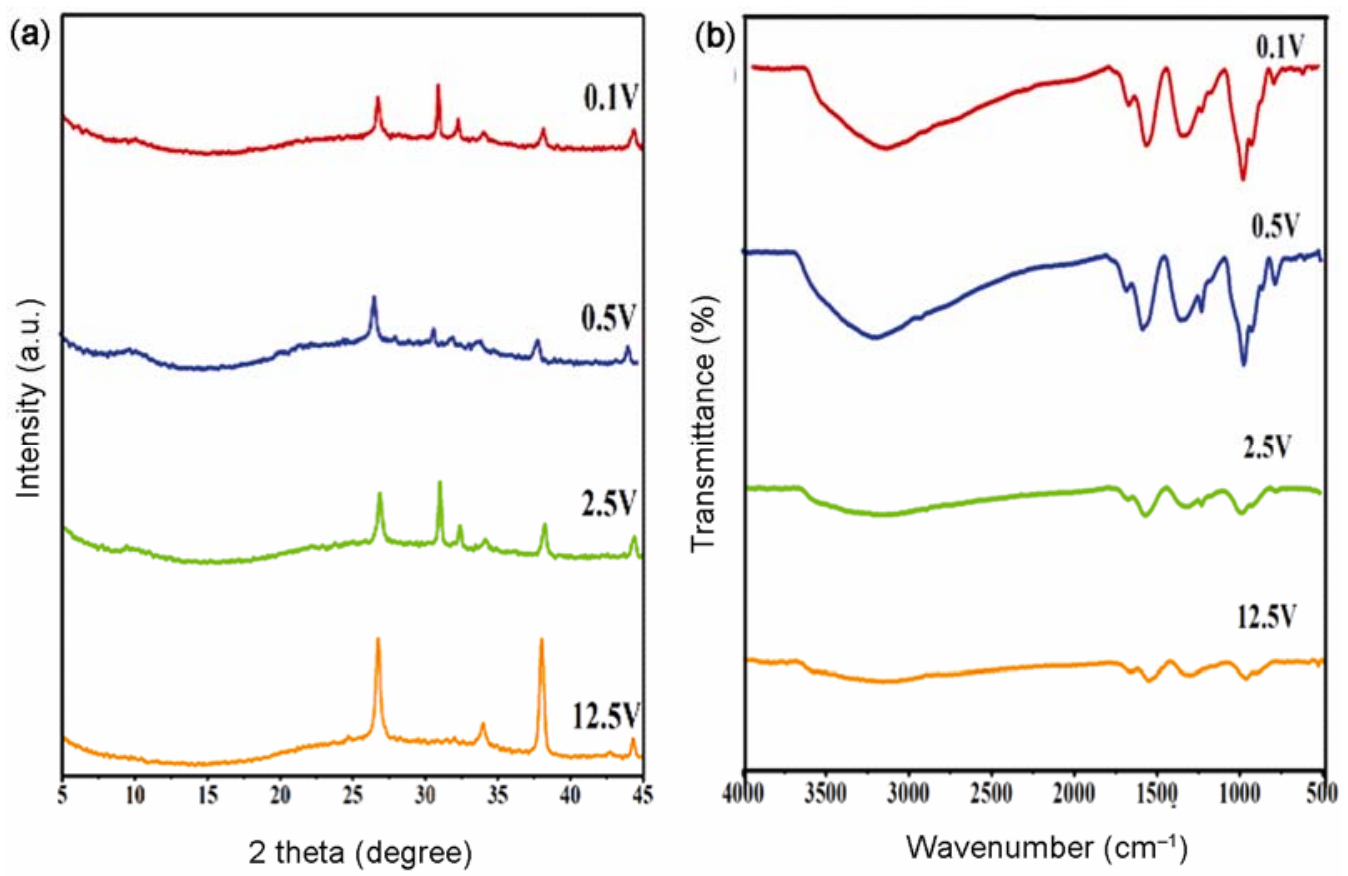

Figure 1. (a) XRD spectra of ERGO obtained from electrochemical reduction in different voltages. (b) FT-IR spectra of ERGO obtained from electrochemical reduction in different voltages. 

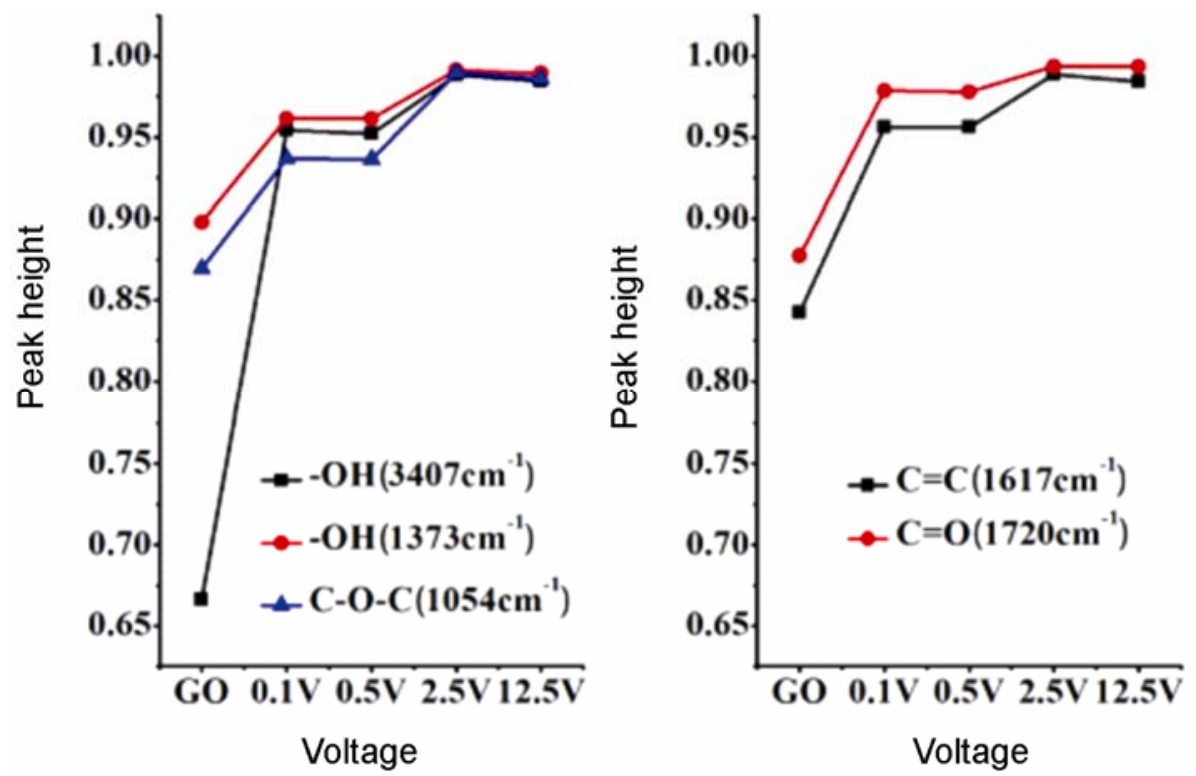

Figure 2. GO and ERGO's absorption heights of chemical groups in FT-IR spectra.

Table 1. FT-IR peak height of chemical groups in different voltages.

\begin{tabular}{lccccc}
\hline & & \multicolumn{5}{c}{ Voltage } \\
\cline { 3 - 6 } Groups & GO & $0.1 \mathrm{~V}$ & $0.5 \mathrm{~V}$ & $2.5 \mathrm{~V}$ & $12.5 \mathrm{~V}$ \\
\hline$-\mathrm{OH}\left(3407 \mathrm{~cm}^{-1}\right)$ & 0.6662 & 0.9544 & 0.9521 & 0.9883 & 0.9847 \\
$-\mathrm{OH}\left(1373 \mathrm{~cm}^{-1}\right)$ & 0.8979 & 0.9617 & 0.9614 & 0.9915 & 0.9893 \\
$\mathrm{C}-\mathrm{O}-\mathrm{C}\left(1054 \mathrm{~cm}^{-1}\right)$ & 0.8697 & 0.9367 & 0.9365 & 0.9897 & 0.9858 \\
$\mathrm{C}=\mathrm{C}\left(1617 \mathrm{~cm}^{-1}\right)$ & 0.8421 & 0.9564 & 0.9562 & 0.9897 & 0.9858 \\
$\mathrm{C}=\mathrm{O}\left(1720 \mathrm{~cm}^{-1}\right)$ & 0.8774 & 0.9786 & 0.9778 & 0.9935 & 0.9936 \\
\hline
\end{tabular}

$0 \cdot 5 \mathrm{~V}$, the characteristic peak of graphite oxide (002) still exist weak absorption nearby $2 \theta=10^{\circ}$ and the characteristic peak of ERGO $\left(2 \theta=26^{\circ}\right)$ is not particularly obvious. On increasing the applied voltage continuity to $2.5 \mathrm{~V}$, the characteristic peak of graphite oxide (002) does not have obvious changes. However, the intensity of characteristic peak of ERGO nearby $2 \theta=26^{\circ}$ increase, which illustrate that crystallinity of the crystals increases and reduction effects become more obvious. When the applied voltage reach to $12.5 \mathrm{~V}$, the characteristic peak of graphite oxide (002) nearby $2 \theta=10^{\circ}$ absolutely disappear and the characteristic peak of ERGO nearby $2 \theta=26^{\circ}$ reach the maximum diffraction intensity, this phenomenon has previously been suggested as partial restoration of the $\pi$ network within the carbon structure and has been witnessed through chemical reduction of GO (Jeong et al 2008; Lei et al 2011). Thus, the intensity of characteristic peak of graphite oxide (002) near $2 \theta=10^{\circ}$ decreases gradually until disappear with an increase of applied voltage. On the contrary, the intensity of the characteristic peak of ERGO nearby $2 \theta=26^{\circ}$ increases gradually.

The spectrum of graphite oxide illustrated $-\mathrm{OH}(v$ (carboxyl)) at $\sim 1373 \mathrm{~cm}^{-1},-\mathrm{OH}($ broad coupling $v(\mathrm{O}-\mathrm{H}))$ at $\sim 3200 \mathrm{~cm}^{-1}$ originated from carboxylic acid, while the band at $\sim 3407 \mathrm{~cm}^{-1}$ could be due to $\mathrm{O}-\mathrm{H}$ stretching mode of intercalated water, $\mathrm{C}=\mathrm{O}(\nu$ (epoxy or alkoxy $))$ at $\sim 1054 \mathrm{~cm}^{-1}, \mathrm{C}=\mathrm{O}$ in carboxylic acid and carbonyl moieties $\left(\gamma\left(\right.\right.$ carbonyl)) at $\sim 1720 \mathrm{~cm}^{-1}, \mathrm{C}=\mathrm{C}$ at $\sim 1617 \mathrm{~cm}^{-1}$ assigned to skeletal vibrations of unoxidized graphitic domains or contribution from the stretching deformation vibration of intercalated water (Jeong et al 2008; Zhang et al 2011). FT-IR spectra of ERGO obtained from electrochemical reduction at different voltages are shown in figure 1(b). After reduction, the height of characteristic peaks variable decrease. In order to clearly illustrate the changes of characteristic peaks of FT-IR spectra of ERGO, the heights of characteristic peaks are calculated with semi-quantitative baseline method (Zhu et al 2011) and listed in table 1. Figure 2 shows influence of voltages on FT-IR peak height of different oxygen-containing groups. Even the applied voltage is very weak, only $0 \cdot 1$ or $0.5 \mathrm{~V}$, GO could be partially reduce with enough time (30 min). Increasing the applied voltage to $2.5 \mathrm{~V}$, the oxygen-containing functional groups in GO are basically disappear and the reduction effects are relatively ideal. Continuing to increase the voltage to $12.5 \mathrm{~V}$, the curve 


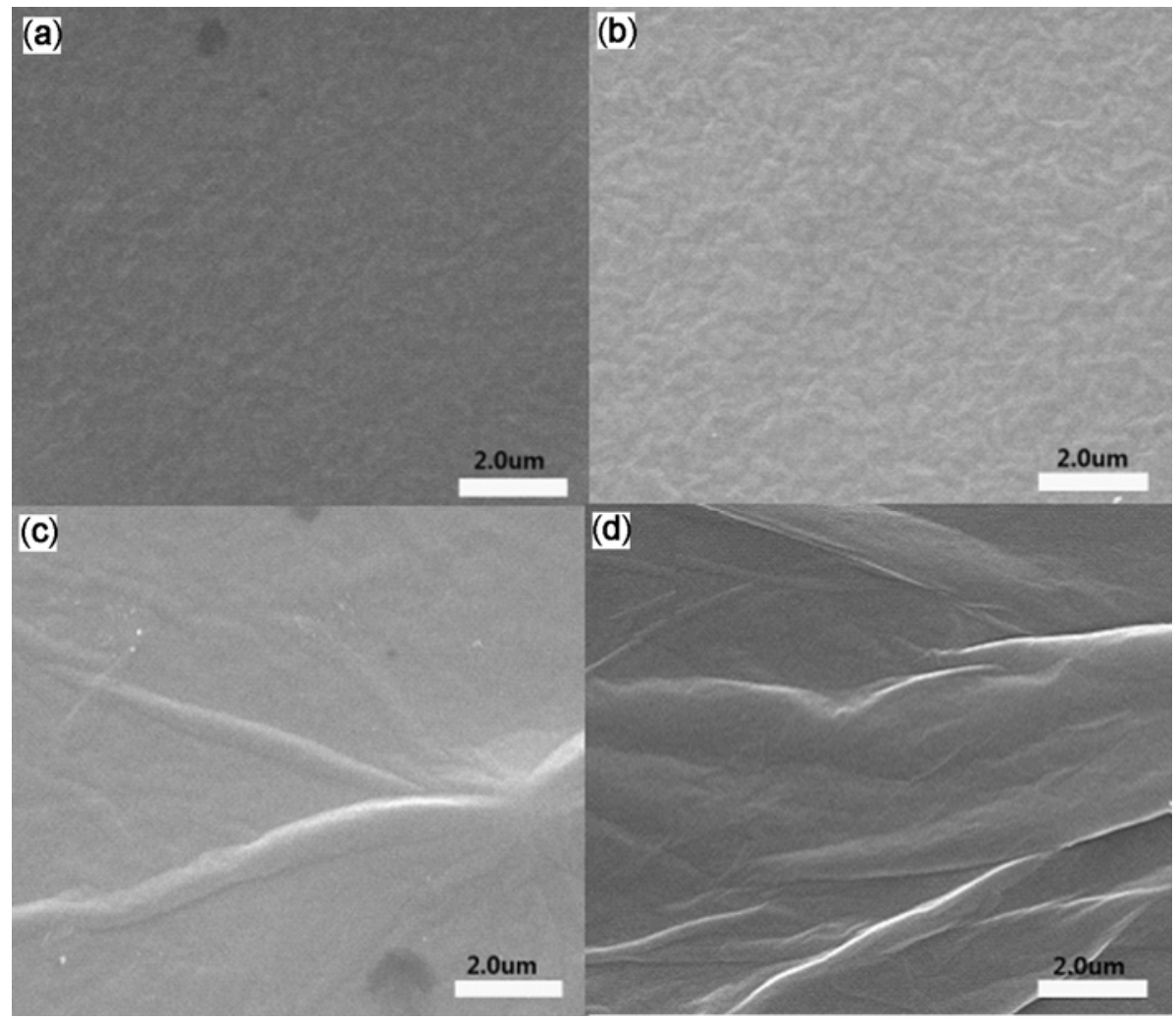

Figure 3. FE-SEM images of ERGO obtained from electrochemical reduction at $0 \cdot 1 \mathrm{~V}$ (a), $0.5 \mathrm{~V}(\mathbf{b}), 2.5 \mathrm{~V}$ (c) and $12.5 \mathrm{~V}(\mathbf{d})$.
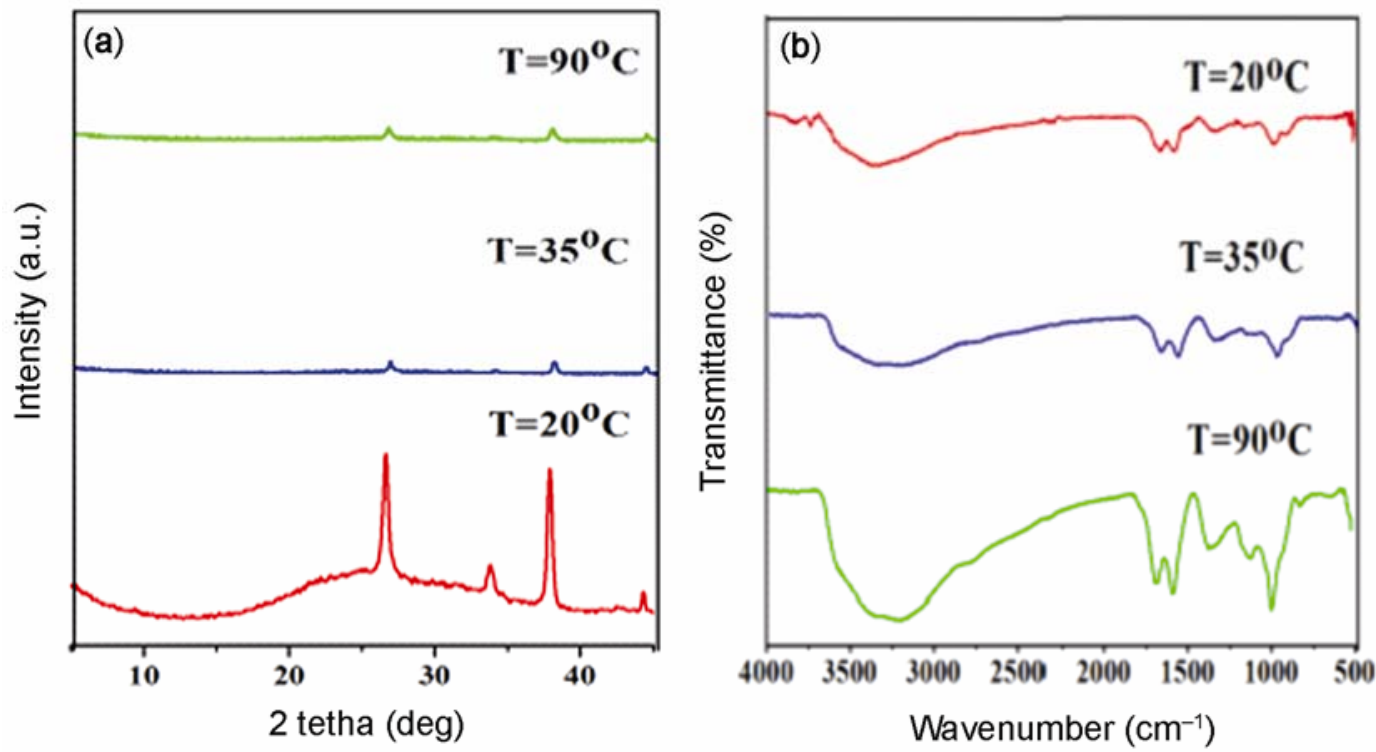

Figure 4. (a) XRD spectra of ERGO obtained from electrochemical reduction in different temperatures. (b) FT-IR spectra of ERGO obtained from electrochemical reduction in different temperatures.

tend to be steady, this show that continuing to increase the applied voltage has little effect on reduction.

FE-SEM images of ERGO obtained from electrochemical reduction at $0.1 \mathrm{~V}(\mathrm{a}), 0.5 \mathrm{~V}(\mathrm{~b}), 2.5 \mathrm{~V}$ (c) and $12.5 \mathrm{~V}$ (d) are showed in figure 3 . The transparency of ERGO films decreases with an increase of voltage, while the wrinkle increases gradually. The corresponding sheet resistance are 22.08, 20.62, 18.88 and $18.28 \Omega \mathrm{sq}^{-1}$, respectively. The sheet resistance decreases gradually with the increase of applied voltage. However, above 

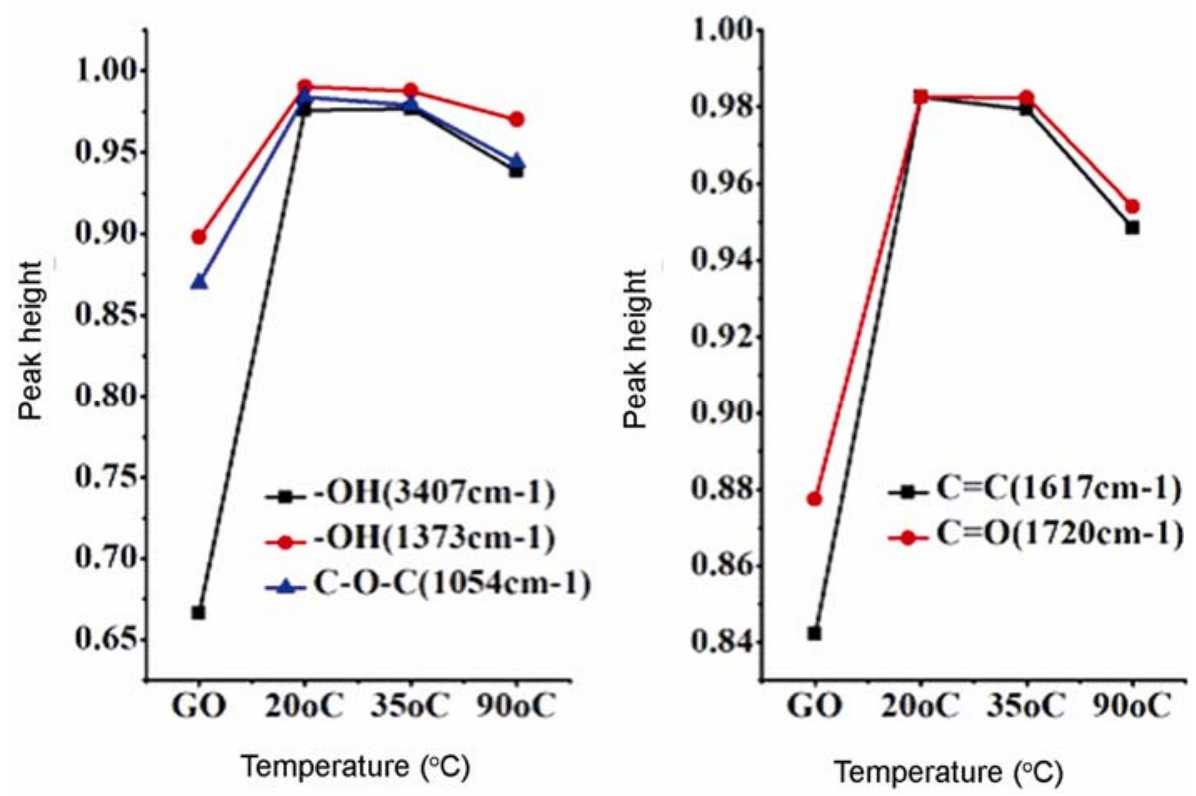

Figure 5. GO and ERGO's absorption heights of chemical groups in FT-IR spectra.

Table 2. FT-IR peak height of chemical groups in different temperatures.

\begin{tabular}{lcccc}
\hline & & \multicolumn{3}{c}{ Temperature } \\
\cline { 3 - 5 } Groups & $\mathrm{GO}$ & $20{ }^{\circ} \mathrm{C}$ & $35{ }^{\circ} \mathrm{C}$ & $90{ }^{\circ} \mathrm{C}$ \\
\hline$-\mathrm{OH}\left(3407 \mathrm{~cm}^{-1}\right)$ & $0 \cdot 6662$ & 0.9756 & 0.9770 & 0.9385 \\
$-\mathrm{OH}\left(1373 \mathrm{~cm}^{-1}\right)$ & $0 \cdot 8979$ & 0.9903 & 0.9877 & 0.9702 \\
$\mathrm{C}-\mathrm{O}-\mathrm{C}\left(1054 \mathrm{~cm}^{-1}\right)$ & $0 \cdot 8697$ & 0.9841 & 0.9791 & 0.9439 \\
$\mathrm{C}=\mathrm{C}\left(1617 \mathrm{~cm}^{-1}\right)$ & $0 \cdot 8421$ & 0.9826 & 0.9793 & 0.9485 \\
$\mathrm{C}=\mathrm{O}\left(1720 \mathrm{~cm}^{-1}\right)$ & $0 \cdot 8774$ & 0.9826 & 0.9824 & 0.9540 \\
\hline
\end{tabular}

$2 \cdot 5 \mathrm{~V}$, increasing voltage has little effect on the sheet resistance of GO films. This result is consistent with XRD and FT-IR analyses. In combination of the analyses result of XRD and FT-IR spectra, the following conclusions can be drawn: as the applied voltage increases from $0 \cdot 1$ to $12 \cdot 5 \mathrm{~V}$, the reduction degree of graphene oxide increases gradually. Above $2.5 \mathrm{~V}$, increasing voltage has little effect on the reduction degree of graphene oxide.

\subsection{Influence of temperature}

During the oxidation process of graphite oxide, at the beginning, temperature increased from 0 to $35^{\circ} \mathrm{C}$ and then reached to $90^{\circ} \mathrm{C}$. Thus, the experiment researched in the applied temperature of 20,35 and $90{ }^{\circ} \mathrm{C}$, respectively. Applied voltage of $2.5 \mathrm{~V}$ was selected. XRD spectra of ERGO obtained from electrochemical reduction at different temperatures are shown in figure 4(a). The sharp diffraction peaks at $2 \theta=\sim 34^{\circ}(101), \sim 38^{\circ}$ (200) come from FTO. When the temperature is $20^{\circ} \mathrm{C}$, the characteristic peaks of GO completely disappear, ERGO reach maximum diffraction intensity. When applied temperature increases from 20 to $35^{\circ} \mathrm{C}$, the intensity of characteristic peak of ERGO nearby $2 \theta=26^{\circ}$ decrease, which illustrate that rising the applied temperature does not increase reduction effect, but weaken it. Continuing to increase the applied temperature to $90{ }^{\circ} \mathrm{C}$, the intensity of characteristic peak of ERGO nearby $2 \theta=26^{\circ}$ further decrease.

FT-IR spectra of ERGO obtained from electrochemical reduction at different temperatures are showed in figure 4(b). After reduction, the height of characteristic peaks decrease to variable degrees. In order to clearly illustrate the changes of characteristic peaks of FT-IR spectra of ERGO, the heights of characteristic peaks are calculated with semi-quantitative baseline method (Zhu et al 2011) and listed in table 2. Figure 5 shows the influence of applied temperature on FT-IR peak height of different oxygen-containing groups. The highest peak appears in FT-IR spectra of ERGO obtained at $20^{\circ} \mathrm{C}$. Comparing to the peak height of $20^{\circ} \mathrm{C}$, the peak heights decrease at 35 and $90^{\circ} \mathrm{C}$.

FE-SEM images of ERGO obtained from electrochemical reduction at $20{ }^{\circ} \mathrm{C}$ (a) $35^{\circ} \mathrm{C}$ (b) and $90{ }^{\circ} \mathrm{C}$ (c) are 


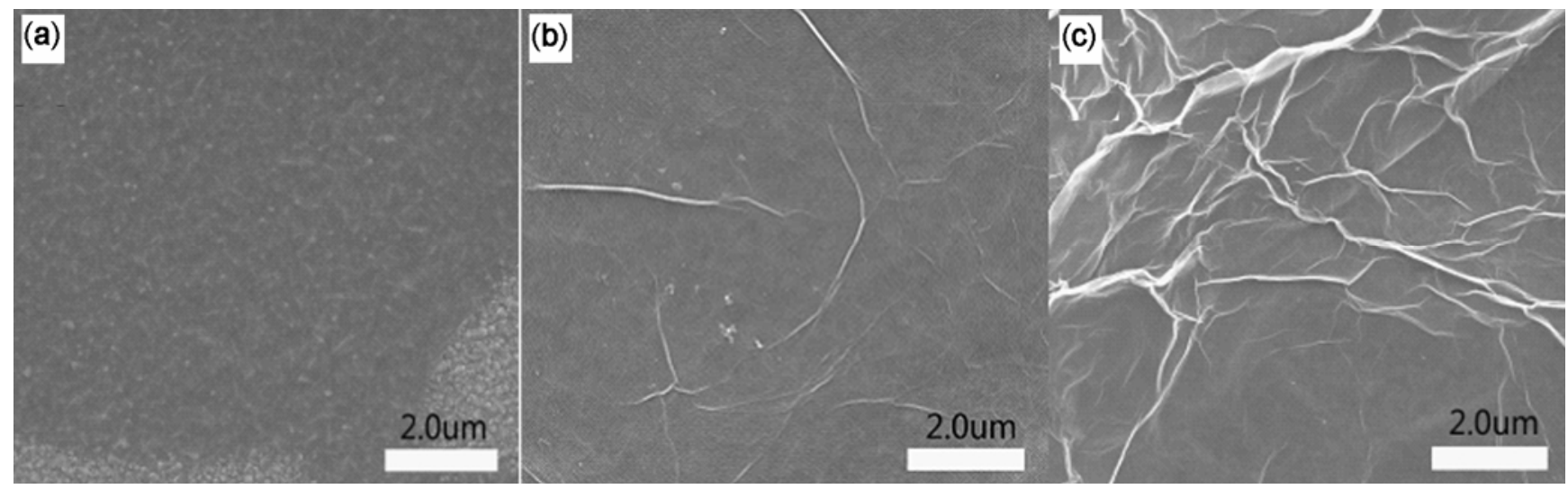

Figure 6. FE-SEM images of ERGO obtained from electrochemical reduction at $20{ }^{\circ} \mathrm{C}(\mathbf{a}), 35{ }^{\circ} \mathrm{C}(\mathbf{b})$ and $90{ }^{\circ} \mathrm{C}(\mathbf{c})$.

showed in figure 6. The transparency of ERGO films decrease with an increase of temperature, while the wrinkle increases gradually. The corresponding sheet resistance are $18.90,19.76$ and $22.08 \Omega \mathrm{sq}^{-1}$, respectively. With the increase of applied temperature, the sheet resistance becomes higher gradually. It also shows that the reduction degree of graphene oxide decreases gradually with the increase of applied temperature. This result is consistent with XRD and FT-IR analyses. In combination of the analysis result of XRD and FT-IR spectra, the following conclusions can be drawn: As the applied temperature increases from 20 to $90{ }^{\circ} \mathrm{C}$, the reduction degree of graphene oxide decreases gradually. The optimal applied temperature found in our experiment is $20^{\circ} \mathrm{C}$.

\section{Conclusions}

(I) Effects of voltage on the electrochemical reduction of graphene oxide: Based on the characterization results of XRD, FT-IR, FE-SEM and four-point probe on the reduced graphene oxide by electrochemical method, as the applied voltage increases from 0.1 to $12.5 \mathrm{~V}$, the reduction degree of graphene oxide increases gradually. Above $2.5 \mathrm{~V}$, increasing applied voltage has little effect on the reduction degree of graphene oxide.

(II) Effects of temperature on the electrochemical reduction of graphene oxide: Based on the characterization results of XRD, FT-IR, FE-SEM and four-point probe on the reduced graphene oxide by electrochemical method, as the applied temperature increases from 20 to $90{ }^{\circ} \mathrm{C}$, the reduction degree of graphene oxide decreases gradually. The optimal applied temperature found in our experiment is $20^{\circ} \mathrm{C}$.

\section{Acknowledgements}

The research in this paper was financially supported by the Programme for Shanghai Basic Research Key Project (12JC1408600) and the National High Technology Research and Development Programme of China (2012AA030303).

\section{References}

Akhavan O and Ghaderi E 2009 J. Phys. Chem. $\mathbf{C 1 1 3}$ 20214

Balandin A A, Ghosh S, Bao W Z, Calizo I, Teweldebrhan D, Miao Feng and Lau C N 2008 Nano Lett. 8902

Buchsteiner A, Lerf A and Pieper J 2006 J. Phys. Chem. B110 22328

Cai W W et al 2008 Science 3211815

Chae H K et al 2004 Nature 427523

Guo H L et al 2009 ACS Nano 32653

Jeong H K et al 2008 J. Am. Chem. Soc. 1301362

Lee C, Wei X and Kysar J W 2008 Science 3213858

Lei Z B, Christov N and Zhao X S 2011 Energy \& Environ. Sci. 261039

Liu S et al 2012 Appl. Surf. Sci. 2585299

Muszynski R B, Seger and Kamat P V 2008 J. Phys. Chem. C112 5263

Sung J A and Yan W Z 2010 J. Phys. Chem. Lett. 11259

Wang Z J et al 2009 J. Phys. Chem. C113 14071

Williams G, Seger B and Kamat P V 2008 ACS Nano 2 1487

Yang J et al 2012 Appl. Surf. Sci. 2585056

Zhang T Y and Zhang D 2011 Bull. Mater. Sci. 3425

Zhu P Y, Zhang D, Xiao S H and Shen M 2011 Phys. BConden. Matter 406498 